\title{
Comparação dos efeitos da eletrolipólise transcutânea e percutânea sobre a gordura localizada na região abdominal e de flancos através da perimetria e análise de bioimpedância elétrica Comparison of lipolysis by transcutaneous and percutaneous electrical stimulation on localized abdominal fat through perimeter and electrical bioimpedance analysis
}

Pâmela Billig Mello, Ft., M.Sc.*, Patrícia Magali Dreher, Ft.**, Aline Martinelli Piccinini, Ft.***, Luís Henrique Telles da Rosa, Ft., D.Sc.****, Patrícia Viana da Rosa, Ft., D.Sc. ${ }^{* * * *}$

*Doutoranda em Ciências Biológicas: Fisiologia - UFRGS, ${ }^{* *}$ Universidade Regional do Noroeste do Estado do Rio Grande do Sul (UNIJUÍ), ***Mestranda em Ciências do Movimento Humano - UFRGS, ****Professor da Universidade Federal de Ciências da Saúde de Porto Alegre/RS (UFCSPA), ****Orientadora do estudo, professora da UNIJUÍ e da Universidade Regional Integrada do Alto Uruguai e das Missöes - URI, campus Erechim

\section{Resumo}

Este estudo buscou verificar a eficácia da utilização da eletrolipólise nos modos transcutâneo e percutâneo no tratamento de adiposidades localizadas na regiấo abdominal e flancos. A amostra foi composta de 18 voluntárias, do gênero feminino, com idades entre 20 e 30 anos. Primeiramente foi realizada uma avaliação clínica, bioimpedância e medidas de perimetria. $\mathrm{O}$ tratamento foi realizado uma vez na semana, totalizando 10 sessóes. A amostra foi dividida em três grupos: Grupo 1, composto de voluntárias que não realizaram tratamento; Grupo 2, tratamento com eletrolipólise no modo transcutâneo (eletrodos de silicone) e Grupo 3, com a utilização do modo percutâneo (agulhas). Após a realização do tratamento as voluntárias foram submetidas à reavaliação. Para a comparação das médias do pré e pós-tratamento em cada grupo foi utilizado o teste tde student. Os resultados do Grupo 1, além de indicarem aumento da gordura corporal, não indicaram diferença nas variáveis de perimetria. Já nos Grupos 2 e 3, apesar de não ter havido diminuição da gordura corporal total, houve diferença significativa na perimetria, parecendo ter ocorrido redução da gordura localizada neste grupo. O estudo demonstrou que a eletrolipólise pode ser usada como um recurso eficaz no tratamento da gordura localizada.

Palavras-chave: fisioterapia, estética, lipólise, gordura abdominal.

\begin{abstract}
This study aimed to evaluate the effectiveness of transcutaneous and percutaneous lipolysis by electrical stimulation in the treatment of localized adiposity in the abdominal region and flanks. The sample consisted of 18 volunteer, female, 20 to 30 years old. We first performed a clinical evaluation, bioelectrical impedance analysis and perimetric measures. The treatment was performed once a week, totaling 10 sessions. The sample was divided into three groups: Group 1 , composed of volunteers who did not receive treatment, Group 2 , treatment with transcutaneous electric stimulation mode (silicon electrodes) and Group 3, with the use of percutaneous stimulation (needle). After the treatment the volunteers were evaluated again. For comparison of pre- and post-treatment in each group was used the Student $t$ test. The results of Group 1 show increasing of body fat and no difference in the variables of perimetry. In Groups 2 and 3, although there has been no decrease in total body fat, was observed significant difference in the perimeter, with reduction of localized fat in this group. The study showed that lipolysis by electric stimulation can be used as an effective therapy in the treatment of localized fat.
\end{abstract} Key-words: physical therapy, esthetics, lipolysis, abdominal fat. 


\section{I ntrodução}

O excesso de gordura corporal e a ação hormonal podem levar ao depósito de tecido gorduroso em determinadas partes do corpo. No homem, o local preferencial é a barriga e na mulher é a região do quadril, onde aparece o culotte. O excesso de gordura pode existir mesmo em pessoas sem excesso de peso, o que explica a presença de culotte mesmo em mulheres aparentemente magras. $\mathrm{O}$ tecido adiposo corresponde a 20 $25 \%$ do peso corporal na mulher e $15-20 \%$ no homem, isso nos casos em que os indivíduos apresentarem peso normal [1].

A hipoderme é a camada mais profunda da pele, conhecida como tela subcutânea, responsável pelo deslizamento da pele sobre as estruturas na qual se apóia. A hipoderme é uma camada rica em tecido adiposo (gordura), distribuída de forma muito irregular pelo corpo [1]. É formada por um grande número de adipócitos. $\mathrm{O}$ adipócito é uma célula que apresenta pequenos vacúolos de gordura distribuídos pelo seu interior [2].

$\mathrm{O}$ excesso de tecido adiposo é um sério problema de saúde, que reduz a expectativa de vida pelo aumento do risco de desenvolvimento de doenças cardíacas coronarianas, hipertensão, hiperdislipidemias, diabetes, osteoartrite e certos tipos de câncer. Para classificar o nível de gordura corporal são recomendados valores de $15 \%$ para homens e $23 \%$ para mulheres, enquanto que a quantidade de gordura de $25 \%$ ou mais para homens e $32 \%$ ou mais para mulheres são considerados como obesidade [3].

Embora as porcentagens de tecido adiposo estejam relacionadas a um fator hormonal sexual, as mulheres mais ativas fisicamente são geralmente as mais magras, este fator é estimulante no momento em que é preciso enfrentar um regime de emagrecimento [3].

No sentido de auxiliar na diminuição da camada de gordura localizada, a Fisioterapia Dermato-Funcional tem proposto algumas técnicas terapêuticas, entre elas está a eletrolipólise [4]. Nesta técnica, aplica-se uma microcorrente específica de baixa frequência (por volta de $20 \mathrm{~Hz}$ ), que atua diretamente nos adipócitos armazenados, produzindo sua desnutriçâo e favorecendo sua posterior eliminação. A eletrolipólise se aplica por meio de finíssimas agulhas implantadas no panículo adiposo. Atualmente se utiliza também a aplicaçâo por intermédio de eletrodos sobre a superfície cutânea, porém, teoricamente, com um efeito mais discreto.

A eletrolipólise consiste na aplicação da corrente elétrica bidirecional com pulsos de baixa frequência, curta duração e componente galvânico nulo, através de pares de eletrodos [5].

O campo elétrico que se origina entre as agulhas ou eletrodo irá provocar algumas modificaçôes fisiológicas locais, que são [4]:

- Aumento da temperatura entre os pólos pela passagem da corrente, o que irá provocar uma vasodilatação e consequentemente um aumento do fluxo sanguíneo, estimulan- do assim o metabolismo celular, facilitando a queima de calorias e a melhora do trofismo celular;

- Movimento iônico, que vai gerar modificaçóes na polaridade da membrana celular;

- Aumento da temperatura local e o efeito direto na parede de vasos de pequeno calibre, que vai gerar uma vasodilatação significativa;

- Estimulação das fibras do tecido conjuntivo subcutâneo, que vai favorecer a drenagem linfática e sanguínea, provocando uma melhora da qualidade e do aspecto da pele;

- Estimulação do sistema simpático, que vai ativar a lipólise; a lipólise ocorre pelo efeito da enzima triglicerídeolipase, que decompôe o triglicerídeo em ácido graxo e glicerol, o ácido graxo é expulso da célula ou é eliminado, o glicerol liberado não pode ser reaproveitado e é captado pelo fígado e metabolizado em glicose.

Outros estudos também sugerem que as fibras dos nervos simpáticos quando ativadas exercem uma influência reguladora sobre o tecido de gordura subcutânea em humanos [6] e em animais [7], assim como a administração de adrenalina ou hormônio adrenocorticotrófico [8].

A prática da eletrolipólise por meio de agulhas requer cuidados de assepsia. O campo elétrico gerado na massa tissular compreendida entre as agulhas altera a permeabilidade da membrana do adipócito aumentando a eliminação dos triglicerídeos intra-adipocitários para o interstício. Essa prática é indolor, e quando surge dor na aplicação normalmente é por mau posicionamento das agulhas que devem permanecer implantadas no tecido subcutâneo dentro do tecido graxo, paralelas entre si [4].

A prática com eletrodos metálicos superficiais geralmente não provoca irritação ou queimadura na pele. Os eletrodos devem ser colocados de acordo com critérios clínicos e do interesse na área a ser tratada [4].

O aparelho de eletrolipólise possibilita o tratamento com várias formas de ondas [9]. Utiliza-se a onda A para diminuir a resistência intrínseca da pele, bem como a sensibilidade dolorosa. A onda B é destinada para uma açáo preferencial na derme, com objetivo de estimular as células, principalmente os fibroblastos na melhora da tonicidade da pele. A onda $\mathrm{C}$ tem como objetivo atuar diretamente nos adipócitos pela estimulação elétrica das terminaçôes do Sistema Nervoso Autônomo Simpático. A ação desta forma de onda se dá diretamente sobre os receptores $\beta$-adrenérgicos que irá desencadear a liberação do AMP cíclico intra-adipocitário, liberando, assim, os ácidos graxos e glicerol. E, por último, as ondas $\mathrm{D}$ e $\mathrm{E}$ que têm ação direta no adipócito e no tecido muscular. A frequência preconizada é de $30 \mathrm{~Hz}$ que promove a eliminação de produtos oriundos da lipólise.

A principal indicação da eletrolipólise está no tratamento da obesidade localizada, celulite e lipodistrofias localizadas, porém existem poucos trabalhos científicos que comprovem a eficácia da técnica em questão [4]. Diante disto e, 
considerando o fato de existirem duas formas diferentes de aplicação desta técnica, o objetivo deste estudo foi verificar e comparar os efeitos da eletrolipólise transcutânea e percutânea sobre a gordura localizada na região abdominal e de flancos, utilizando, para isso, medidas perimétricas e análise de bioimpedância elétrica.

\section{Material e métodos}

\section{Caracterização da pesquisa}

Este estudo se caracteriza por ser do tipo experimental, através da comparação entre amostras independentes, com uma abordagem quantitativa.

\section{Local do estudo}

Esta pesquisa foi realizada no município de Ijuí/RS, nas dependências da Clínica Escola da Universidade Regional do Noroeste do Estado do Rio Grande do Sul - UNIJUÍ.

\section{Aspectos éticos}

Para a efetivação da presente pesquisa o projeto de pesquisa foi encaminhado ao Comitê de Ética em Pesquisa da Universidade Regional Integrada do Alto Uruguai e das Missóes - URI, campus Erechim, e aprovado sob o parecer consubstanciado 134/TCH/06.

Para realização da coleta de dados foi solicitada autorização dos participantes do estudo, por meio do Termo de Consentimento Livre e Esclarecido, conforme resolução 196/96 do Conselho Nacional de Saúde, a qual trata das Diretrizes e Normas Regulamentadoras de Pesquisa Envolvendo Seres Humanos.

A coleta dos dados ocorreu somente após a autorização, a concordância e a assinatura do termo de consentimento pelos participantes.

\section{População e amostra}

A população-alvo deste estudo foi constituída por voluntárias do gênero feminino, com idades entre 20 e 30 anos.

A amostra do estudo foi de 18 sujeitos, divididos em três grupos: Grupo1, grupo controle, sem utilizar tratamento; Grupo 2, utilizando a forma transcutânea de eletrolipólise; e, Grupo 3, utilizando a forma percutânea.

Todos foram selecionados de forma intencional por voluntariedade. Foram adotados como critério de exclusão da amostra: ter idade inferior a 20 e superior a 30 anos; possuir doenças crônicas, próteses metálicas ou marcapasso cardíaco; possuir feridas abertas e/ou processo inflamatório na região ou perto da região tratada; e demais contra-indicaçôes clássicas para o uso de aparatos de eletroterapia [5,9].

\section{Instrumento de avaliação}

Utilizamos os seguintes instrumentos para avaliação dos resultados:

- Perimetria: Foi avaliada a medida da circunferência abdominal, com a utilização de uma fita métrica, em três regiōes corporais: região supraumbilical $(5 \mathrm{~cm}$ acima da cicatriz umbilical), regiâo da cicatriz umbilical (sobre a cicatriz umbilical) e regiáo infraumbilical $(5 \mathrm{~cm}$ abaixo da cicatriz umbilical [10].

- Bioimpedância elétrica: método para avaliar a composição corporal, sendo utilizada para determinar a água corporal e estimar a massa livre de gordura e o percentual de gordura. Para tal foi utilizado um equipamento analisador de Bioimpedência - Modelo de Mesa da Marca CompCorp Sistemas, sendo esta medida realizada na Clínica de Nutrição da UNIJUÍ. O teste foi realizado no lado dominante do indivíduo com o mesmo em posição supina, os braços e pernas foram separados em aproximadamente 30 graus a partir de uma linha média. Os locais de colocaçáo de eletrodos foram limpos com álcool e as jóias usadas retiradas. Um eletrodo foi colocado na mão e outro no pé. Após a conexáo dos cabos aos eletrodos, registrou-se os valores correspondentes [11].

\section{Procedimentos}

Inicialmente foi feito um contato com os interessados em participar do estudo. As voluntárias assinaram Termo de Consentimento Livre e Esclarecido, o qual informou sobre os objetivos do estudo e informava que o tratamento não apresentava risco à saúde das participantes e que elas poderiam desistir de participar a qualquer momento sem mais implicações.

$\mathrm{Na}$ primeira sessão foi realizada a medida da perimetria e a avaliação por bioimpedância de acordo com o descrito previamente.

O tratamento foi realizado utilizando o aparelho HVS Eletrolipólise da marca KW Indústria Nacional de Tecnologia Eletrônica Ltda, nos modos Transcutâneo e Percutâneo.

Para a modalidade transcutânea utilizamos eletrodos que foram colocados em um espaço máximo de $5 \mathrm{~cm}$ entre eles, com o gel para a condução da corrente. O estímulo é transmitido através da corrente elétrica bidirecional com pulsos de baixa frequência, curta duração e componente galvânico nulo através de pares de eletrodos.

Para o uso do modo percutâneo, inicialmente precederam cuidados de assepsia. As agulhas foram introduzidas na posição vertical logo abaixo da pele, na junçâo dermehipoderme. Essa prática é indolor, e quando surge dor na aplicação normalmente é por mau posicionamento das agulhas, neste caso as agulhas eram retiradas e introduzidas novamente, até o momento em que a voluntária não relatasse mais dor. 
A frequência escolhida tanto para o tratamento na forma percutânea quanto transcutânea foi de $30 \mathrm{~Hz}$ associado à forma de onda C. A intensidade foi alterada de acordo com a sensibilidade de cada voluntária. As sessōes foram realizadas uma vez por semana, com duração de 50 minutos cada sessão, durante 10 semanas. Ao final do tratamento foi realizada uma reavaliação.

\section{Análise dos resultados}

Os dados coletados foram analisados com a utilização da estatística descritiva através do uso do programa Microsoft Excel e SPSS, versão 11.1.

As variáveis quantitativas foram descritas em função de sua média e desvio padrão (média $\pm \mathrm{DP}$ ).

Para a comparação das médias em cada grupo, antes e depois do tratamento, foi utilizado o Teste de $t$ de student para amostras dependentes, com intervalo de confiança de $95 \%$.

Para a comparação entre os três grupos foi utilizada a Análise de Variança (ANOVA) de uma via seguida de post hoc apropriado, quando necessário, com intervalo de $95 \%$.

\section{Resultados}

A amostra do estudo foi homogênea. A idade mínima e máxima das voluntárias foi de 20 e 30 anos, respectivamente, com uma média de 23,22 ( \pm 3,04) anos. Na comparação entre os Grupos 1, 2 e 3 não foi identificado diferença significativa na idade, sendo a média de idade 24,67 $( \pm 4,45)$, $21,67( \pm 1,36)$ e $23,33( \pm 2,06)$ anos para os grupos 1,2 e 3 , respectivamente.

Os resultados do IMC indicam um valor mínimo de 18 e máximo de 25 , sendo a média $21,60( \pm 1,95)$ e o valor dos grupos 1,2 e $323,04( \pm 1,76), 20,75( \pm 1,74)$ e $21,01( \pm 1,75)$ $\mathrm{kg} / \mathrm{m}^{2}$, respectivamente. De acordo com as categorizaçôes do IMC para este valor, estes sujeitos podem ser classificados dentro de uma faixa de normalidade [10]. Na comparação entre o IMC dos Grupos 1, 2 e 3 não foi identificada diferença significativa, mostrando mais uma vez o homogeneidade da amostra.

Os resultados da análise de bioimpedância elétrica do Grupo 1 (controle) indicam haver uma diferença significativa entre as variáveis gordura corporal e massa magra, havendo um aumento da gordura corporal ao final do período de tratamento. Com relação à massa magra final houve uma diminuição em relação aos valores iniciais. As demais variáveis, inclusive as medidas perimétricas, não apresentaram diferenças significativas (Tabela I).

O Grupo 2 recebeu tratamento de eletrolipólise no modo transcutâneo. Na comparaçáo entre os valores antes e depois do tratamento na Análise de Bioimpedância Elétrica no Grupo 2 náo foi identificada diferença significativa na gordura corporal e na massa magra. Foi observada diferença entre a água corporal inicial e final (Tabela II).

Nas medidas perimétricas foi observada uma redução de todas as medidas (Tabela II).

O Grupo 3 recebeu tratamento com a utilização da técnica percutânea, através do uso de agulhas intra-hipodérmicas. Neste grupo foi encontrada diferença significativa entre algumas variáveis mensuradas através da Análise de Bioimpedância Elétrica: Gordura Corporal (que aumentou), Massa Magra (que diminuiu), e Água Corporal (que diminuiu; Tabela III).

Nas medidas perimétricas, apenas a perimetria umbilical diminuiu significativamente (Tabela III).

$\mathrm{Na}$ comparação entre os Grupos 1, 2 e 3 não se observou diferença significativa entre as variáveis analisadas.

\section{Discussão}

Os resultados da Análise de Bioimpedância Elétrica do Grupo 1 (controle) indicam haver uma diferença significativa entre as variáveis Gordura Corporal e Massa Magra, havendo um aumento da Gordura Corporal em relaçáo à Gordura Corporal Inicial. Com relação à Massa Magra houve uma diminuição dos valores iniciais. Este resultado era esperado, uma vez que o acúmulo de gordura corporal no gênero feminino

Tabela I - Descrição dos resultados da análise de bioimpedância elétrica e medidas perimétricas do Grupo 1.

\begin{tabular}{lcccc}
\hline & Variáveis & Média & Desvio padrão & Teste t de student \\
\hline Gordura & Inicial & 25,50 & 4,37 & \\
Corporal & Final & 27,83 & 3,18 & $0,04^{*}$ \\
\hline Massa & Inicial & 74,5 & 4,37 & $0,04^{*}$ \\
Magra & Final & 72,17 & 3,18 & \\
\hline Água & Inicial & 56 & 4,56 & 0,15 \\
Corporal & Final & 52,83 & 2,48 & 0,51 \\
\hline Perimetria & Inicial & 71,25 & 2,60 & \\
Supraumbilical & Final & 70,50 & 3,71 & 0,92 \\
\hline Perimetria & Inicial & 78,42 & 2,2 & 0,10 \\
Umbilical & Final & 78,33 & 2,73 & 1,68 \\
\hline Perimetria & Inicial & 82,58 & 1,9 & \\
Infraumbilical & Final & 82,92 & &
\end{tabular}

$N=6,{ }^{*} p \leq 0,05$. 
Tabela II - Descrição dos resultados da Análise de Bioimpedância Elétrica e medidas perimétricas do Grupo 2.

\begin{tabular}{lcccc}
\hline & Variáveis & Média & Desvio Padrão & Teste + de student \\
\hline Gordura & Inicial & 23,17 & 5,98 & \\
Corporal & Final & 24,67 & 4,36 & 0,09 \\
\hline Massa & Inicial & 76,83 & 5,98 & 0,09 \\
Magra & Final & 75,33 & 4,36 & $0,01^{*}$ \\
\hline Água & Inicial & 56,50 & 4,32 & \\
Corporal & Final & 55,00 & 3,34 & $0,01^{*}$ \\
\hline Perimetria & Inicial & 73,17 & 4,20 & $0,01^{*}$ \\
Supraumbilical & Final & 70,08 & 4,58 & \\
\hline Perimetria & Inicial & 77,75 & 5,00 & $0,03^{*}$ \\
Umbilical & Final & 74,42 & 4,65 & 4,97 \\
\hline Perimetria & Inicial & 82,17 & 4,35 & \\
Infraumbilical & Final & 79,17 & &
\end{tabular}

$N=6,{ }^{*} p \leq 0,05$.

Tabela III - Descriçâo dos resultados da Análise de Bioimpedância Elétrica e medidas perimétricas do Grupo 3.

\begin{tabular}{lcccc}
\hline & Variáveis & Média & Desvio padrão & Teste t de student \\
\hline Gordura & Inicial & 24,83 & 1,72 & \\
Corporal & Final & 26,83 & 2,13 & $0,02^{*}$ \\
\hline Massa & Inicial & 75,17 & 1,72 & $0,02^{*}$ \\
Magra & Final & 73,17 & 2,13 & \\
\hline Água & Inicial & 55,00 & 1,26 & $0,04^{*}$ \\
Corporal & Final & 53,67 & 1,75 & 0,07 \\
\hline Perimetria & Inicial & 71,58 & 5,22 & $0,01^{*}$ \\
Supraumbilical & Final & 68,92 & 3,52 & 0,08 \\
\hline Perimetria & Inicial & 78,83 & 4,50 & 0,58 \\
Umbilical & Final & 75,08 & 3,76 &
\end{tabular}

$N=6,{ }^{*} p \leq 0,05$.

pós-puberdade é normal, segundo Guedes \& Guedes [12]. Porém, intervençôes que busquem modificar esta característica corporal devem ser baseadas em estratégias que podem ser diversas: alimentação, exercício, tratamentos estéticos etc [2]. Desta forma, esperar alteraçóes que conduzam à redução da gordura corporal sem nenhum tipo de intervenção parece ser muito limitado.

No grupo tratado com eletrolipólise no modo transcutâneo não ocorreu diminuição da gordura corporal total, isto pode ser devido à ação deste tratamento ser voltada à gordura localizada. A principal indicação da eletrolipólise está no tratamento da obesidade localizada, celulite e lipodistrofias localizadas [4]. Isto limitaria a possibilidade de haver alteraçôes na composição corporal total.

Porém, Paula et al. [13] demonstraram que, além de reduzir as medidas perimétricas de abdômen, a eletrolipólise pode exercer um efeito na mobilização dos lipídios, reduzindo as concentraçôes séricas de glicerol após seis sessôes.

A aplicação da eletrolipólise por intermédio de eletrodos sobre a superfície cutânea demonstra um efeito mais discreto [4]. Porém, as diferenças encontradas nas medidas perimétricas indicam que houve uma redução do perímetro supraum- bilical, umbilical e infraumbilical, podendo sim esta ter sido causado pela ação do tratamento desenvolvido.

Em relaçáo a isso, Garcia et al. [14] estudaram a aplicação de eletrolipólise na correção de assimetrias corporais pós-lipoaspiração abdominal e avaliaram seus resultados por fotografias e ressonância magnéticas nuclear, obtendo uma redução de 19,86\% do tecido adiposo da regiáo tratada.

Ainda, Chein et al. [15] verificaram que a estimulação elétrica transcutânea de acupontos modifica a composição corporal em mulheres obesas na pós-menopausa, diminuindo as medidas de circunferência de cintura e o percentual de gordura corporal.

No Grupo 3 houve um aumento na Gordura Corporal e uma diminuição da Massa Magra. Por outro lado, embora não tenha ocorrido uma diferença significativa houve uma diminuição nos valores das medidas de perimetria supraumbilical e infraumbilical, e uma diminuição significativa da perimetria umbilical. Estes resultados podem ter sido influenciados por outras variáveis, especialmente no aspecto nutricional, uma vez que este não foi controlado durante a pesquisa.

Embora alguns autores [4], considerem que a eletrolipólise apresenta maiores resultados através do modo percutâneo 
com a aplicação com agulhas, nossos resultados apontaram para diminuiçóes de gordura abdominal mais significativas no grupo tratado de forma transcutânea, o que pode ser um fator positivo, uma vez que esta técnica não é invasiva e de mais fácil aplicação.

\section{Conclusão}

Este estudo teve como principal objetivo comparar os efeitos da eletrolipólise transcutânea e percutânea no tratamento da gordura localizada na regiáo abdominal e de flancos. Os resultados do estudo permitiram identificar que ambas as técnicas possibilitam a redução das medidas perimétricas que mensuram a gordura localizada nestas regiōes; porém, a avaliação da composição corporal total mostrou que a eletrolipólise, ao menos sozinha, não é capaz de modificar a gordura corporal total.

Estudos adicionais deveriam ser realizados com grupos maiores e com um controle dos aspectos nutricionais e da atividade física.

\section{Referências}

1. Junqueira C. Histologia básica. 8a ed. Rio de Janeiro: Guanabara Koogan; 1995.

2. Guirro E, Guirro R. Fisioterapia dermato-funcional. $3^{\mathrm{a}} \mathrm{d}$. Barueri: Manole; 2002.

3. Nardeu M, Pérronet F. Fisiologia aplicada na atividade física. São Paulo: Manole; 1985.
4. Ullmann D, Reis TM, Steibel V. Curso de atualização em medicina estética. 4a ed. Porto Alegre: [s.ed.]; 2003.

5. Busnardo VL. Eletrolipólise - Eletrolipoforese. [s.ed.]; 2003.

6. Dodt C, Lonnroth P, Fehm HL, Elam M. Intraneural stimulation elicits an increase in subcutaneous interstitial glycerol levels in humans. J Physiol 1999;521:545-52.

7. Chan TM, Dehave JP, Tatoyan A. Activation of lipolysis by epinephrine and electrical stimulation in the perfused hindquarters of lean and obese-diabetic (DB/DB) mice. Biochim Biophys Acta 1983;751(3):384-92.

8. Kumon A, Hara T, Takashashi A. Effects of catecholamines on the lipolysis of two kinds of fat cells from adult rabbit. J Lipid Res 1976;17(6):559-64.

9. Campos MSP. Manual do aparelho HVS eletrolipólise. [s.ed.]; 2000.

10. Leite PF. Aptidão física, esporte e saúde: prevenção e reabilitação. 2a ed. Sáo Paulo: Robe; 1990.

11. Manual de avaliação corporal por bioimpedância - Guia de Introdução. [s.ed.]; [s.d.].

12. Guedes DP, Guedes JERP. Composição corporal: princípios, técnicas e aplicaçóes. Londrina: CEITEC; 2003.

13. Paula MR, Picheth G, Simóes NDP. Efeitos da eletrolipoforese nas concentraçóes séricas do glicerol e do perfil lipídico. Fisioter Bras 2007; Suplemento Especial: 5-10.

14. Garcia PG, Garcia FG, Borges FS. O uso da eletrolipólise na correção de assimetria no contorno corporal pós-lipoaspiração: relato de caso. Revista Fisioterapia Ser 2006;1(4):1-9.

15. Chien LW, Lin MH, Chung HY, Liu CF. Trancutaneus electrical stimulation of acupoints changes body composition and heart rate variability in postmenopausal women with obesity. Evid Based Complement Alternat Med 2009. 